\title{
EL DEBATE COMPETITIVO EN EL AULA COMO TÉCNICA DE APRENDIZAJE COOPERATIVO EN LA ENSEÑANZA DE LA ASIGNATURA DE RECURSOS HUMANOS
}

\section{Competitive debate classroom as a cooperative learning technique for the human resources subject}

\author{
Guillermo A. SÁnchez Prieto \\ Departamento de Gestión Empresarial \\ Facultad de Ciencias Económicas y Empresariales \\ Universidad Pontificia Comillas de Madrid \\ Correo-e: gsprieto@comillas.edu
}

Recepción: 6 de octubre de 2015

Envío a informantes: 18 de octubre de 2015

Aceptación definitiva: 7 de noviembre de 2015

Resumen: El artículo expone el debate académico de competición como una técnica de aprendizaje cooperativo para la enseñanza de la asignatura de recursos humanos en el ámbito de los grados universitarios. El objetivo general de este trabajo es comprobar si se puede incluir esta técnica en el abanico del aprendizaje cooperativo. El objetivo específico consiste en presentar un modelo con el que llevar a cabo esta técnica en la enseñanza de los recursos humanos. Así, la primera parte del artículo abarca el concepto de aprendizaje cooperativo y sus características fundamentales. La segunda parte presenta el modelo de debate más apto para los criterios del concepto de aprendizaje cooperativo. La tercera parte concluye con las características del modelo de debate que cumplen distintos aspectos o no del aprendizaje cooperativo de cara a la asignatura de recursos humanos.

Palabras clave: debate; aprendizaje cooperativo; oratoria; argumentación; recursos humanos.

Aвstract: The paper shows an academic debate model as a cooperative learning technique for teaching human resources at University. The general objective of this paper is to conclude if academic debate can be included in the category of cooperative learning. The Specific objective it is presenting a model to implement this technique. Thus the first part of the paper shows the concept of cooperative learning and its main 


\section{4 \\ EL DEBATE COMPETITIVO EN EL AULA COMO TÉCNICA DE APRENDIZAJE COOPERATIVO EN LA ENSEÑANZA DE LA ASIGNATURA DE RECURSOS HUMANOS GUILLERMO A. SÁNCHEZ PRIETO}

characteristics. The second part presents the debate model believed to be labelled as cooperative learning. Last part concludes with the characteristics of the model that match different aspects or not of the cooperative learning.

KEY WORDs: debate; cooperative learning; public speaking; argumentation; human resources.

\section{Introducción}

$\mathrm{E}$ STE ARTÍ́CULO PROPONE UN MODELO DE APRENDIZAJE COOPERATIVO: el debate competitivo en el aula como técnica de aprendizaje cooperativo en el entorno educativo.

Así, el presente trabajo expondrá qué es el aprendizaje cooperativo y cuáles son sus características fundamentales. En segundo lugar se expondrá el modelo de debate académico que ya se está llevando a cabo en distintos centros educativos universitarios y no universitarios. Como tercera parte, se observará qué características cumple el modelo propuesto respecto a las metodologías activas y especialmente al aprendizaje cooperativo.

En el ámbito universitario es donde el debate ha tenido mejor acogida. Sólo en la Comunidad de Madrid se puede participar en más de I2 torneos en un mismo año académico a fecha de hoy (mayo de 20I7).

\section{Experiencias universitarias de debate en el aula en España}

Disponemos de pocas experiencias universitarias contrastadas sobre debate en el aula en España. El autor de este artículo ha llevado a cabo experimentos con alumnos de la Universidad Pontificia Comillas de Madrid en la asignatura Habilidades Profesionales para las carreras de Ciencias Políticas y Derecho. En la misma Universidad, la profesora de Derecho Internacional Público Irene Quintans emplea también dicha técnica. En su asignatura los alumnos estudian parte del temario mediante la técnica del debate competitivo y con la misma son evaluados.

En lo estrictamente académico existen asignaturas de debate. En España desde 2005 se imparten asignaturas de debate en las Universidades Pontificia Comillas de Madrid, de Salamanca o de Almería, entre otras. En el ámbito internacional, al margen de competiciones, el tratamiento científico del debate se ha trabajado con exhaustividad. Se han escrito artículos sobre la aplicación del debate en ámbitos tan dispares como el derecho, la enfermería, la ingeniería o la odontología (Abhijit y Macchiette, 2005; Bellon, 2000; Mitchell, 1998; Takanokura y Masato, 2008; Yasuko, 2003).

Así, existen congresos científicos y cátedras de debate. También hay journals sobre la materia como el Contemporary Argumentation and Debate, Argumentation and Advocay o el Journal of the Communication, Speech E Theatre Association of North Dakota.

Se han llevado a cabo experiencias de formación para el profesorado en esta técnica. El autor de este artículo ha implementado formaciones sobre el uso del debate como herramienta de enseñanza y aprendizaje en la Universidad Europea de Madrid. 
En lo referente al ámbito escolar se han impartido formaciones sobre el uso del debate en el aula en los centros escolares Balder, Everest, Inmaculada de Gijón, Estudio en Madrid y San Ignacio de Oviedo. Sin embargo, hasta la fecha no se dispone de bibliografía científica sobre el uso del debate en el aula en España. Como opción más clara de debate se maneja el manual de Oscar Brenifier Enseñar mediante el debate. Dicho manual tampoco especifica qué se entiende por debate y se utilizan indistintamente términos como debate o discusión en la versión francesa (Brenifier, 2005: 35). No obstante, en algo sí se coincide con Brenifier y es que «a través de una formalización y depuración de la técnica del debate [...]. Se puede conseguir esa cuadratura del círculo en que los niños filosofen entre ellos a través de una formalización y depuración de la técnica del debate» (Brenifier, 2005: I4).

El propio Brenifier (2005: I4) manifiesta que lo que conocemos como debate argumentado (debate competitivo en nuestro modelo), propio de países anglosajones, «es difícil que este tipo de ejercicio puede ser aplicado a educación primaria, es más propio de educación secundaria, y se le está reservando a cursos superiores». Desde nuestra experiencia, este tipo de debate es perfectamente aplicable en las aulas de educación primaria, como reveló el debate llevado a cabo en el colegio público Julián Marías de Madrid con estudiantes de primaria. En este caso se adoptó una resolución de debate propia de este ciclo educativo, como fue: «Los mayores del Instituto son malos».

El artículo se divide en tres partes. La primera explicará en qué consiste el modelo de debate en el aula antes, durante y después de un debate para la enseñanza de recursos humanos. En segundo lugar, se hará ver cuáles son las consecuencias del debate con las metodologías activas y en concreto con el aprendizaje cooperativo.

\section{Metodología}

\section{I. Objetivos que persigue la investigación}

- Discernir si el debate competitivo en el aula puede ser concebido como una técnica per se.

- Averiguar si la técnica de debate competitivo en el aula está comprendida dentro de las metodologías activas.

- Establecer si el debate en el aula está comprendido entre las técnicas de aprendizaje cooperativo.

\subsection{Aplicación más allá de conocimiento científico}

Como se ha expuesto al principio del artículo, esta técnica de debate en el aula se lleva aplicando de manera competitiva y lúdica en varios ámbitos académicos en España. Sin embargo, en este trabajo pretendemos sistematizar y dotar de rigor conceptual a dicha técnica.

Actualmente, en España y en otros países se empieza utilizar el debate y se sospechan, aunque no confirman, sus aplicaciones en el aula. Este trabajo pretende comenzar a solucionar la escasez de rigor pedagógico a la hora de emplear el debate en el aula. 


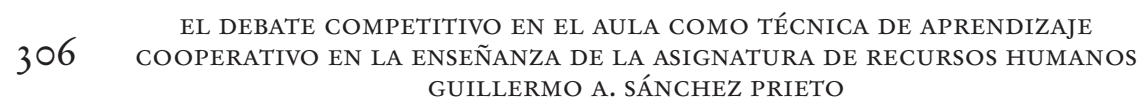

3.3. Naturaleza de la investigación y tipo de metodología usada

Este trabajo es de naturaleza bibliográfica y conceptual. No se pretendía experimentar, ni se consideraba necesario para alcanzar los objetivos del trabajo. La metodología de investigación consistió en los siguientes pasos:

a) Recopilación y acopio de fuentes sobre aprendizaje cooperativo y metodologías activas. En esta fase se extrajeron y analizaron los distintos conceptos según distintos autores de metodología activa y de aprendizaje cooperativo.

b) Extracción de características comunes y particulares de cada una de las definiciones de aprendizaje cooperativo. A la luz de los distintos autores que manejan el concepto de aprendizaje cooperativo, se extrajeron sus características comunes y particulares.

c) Por último, se extrajeron las afinidades del debate con los conceptos de aprendizaje cooperativo y los conceptos de metodologías activas.

\section{El concepto de debate y el modelo de debate en el aula}

Antes de explicar en qué consiste nuestro modelo de debate se hace necesario definir qué se entiende por debate.

Adelino Cattani (2003: 67) define el debate como: «Una competición (un reto, un desafío) entre dos antagonistas, en los que, a diferencia de lo que ocurre en una simple discusión, existe una tercera parte (un juez, un auditorio) cuya aprobación buscan los dos contendientes. Se puede debatir, incluso sobre cuestiones que se consideran imposibles de resolver con el objetivo de persuadir a otros». Se destaca un elemento distintivo, el de la tercera parte, juez o auditorio y su aprobación. En una discusión cotidiana sobre cualquier tema intentamos convencer a nuestro interlocutor. No ocurre así en un debate académico en el que se debe convencer al juez o jurado.

El exprofesor de debate y comunicación personal de la Universidad Santa María de Texas Alan Cirlin no ofrece una definición explícita de qué es un debate, pero lo diferencia del concepto de argumentación con fines persuasivos: «Argumentamos con alguien para convencerle y debatimos contra un oponente para convencer a un auditorio» (Cirlin, 1999: 8). Una vez más se distingue la presencia de una tercera parte con un poder decisorio.

Huber, por su parte, menciona que «es el proceso de presentación de argumentos A favor o En contra de una proposición» (2007: 7). No añade más, aunque queda claro que ha de haber un lado A favor y otro En contra de la cuestión y que hay un proceso de presentación.

Para comprender bien las dinámicas que se generan en un debate es necesario conocer la secuencia de acciones que ha de seguir un docente para conducir un debate en el aula como herramienta de aprendizaje según nuestro modelo. Establecemos como ejes vertebradores las acciones a llevar a cabo antes, durante y después del debate. Asimismo, intercalamos las acciones que de manera simultánea o paralela han de seguir docentes y alumnos. Los pasos que se verán a continuación no tienen un máximo de tiempo, a excepción del formato del debate como tal. Las tareas se pueden hacer tanto en el aula como fuera de ella, algunas on-line. 
EL DEBATE COMPETITIVO EN EL AULA COMO TÉCNICA DE APRENDIZAJE COOPERATIVO EN LA ENSEÑANZA DE LA ASIGNATURA DE RECURSOS HUMANOS GUILLERMO A. SÁNCHEZ PRIETO

\section{I. Pasos previos al debate}

El origen de este modelo está basado en las competiciones de debate universitario. No obstante, una aplicación pedagógica en el contexto universitario en aula se llevó a cabo a modo de experimento en la asignatura de Instituciones de derecho comunitario que impartía la profesora Irene Quintans en la Universidad Pontificia Comillas de Madrid. Dichos resultados se presentaron en el Congreso de Innovacion Docente de la Universidad Europea de Madrid en el 2007.

A continuación, se estudiará qué pasos han de seguir tanto docentes como alumnos a la hora de preparar un debate en el contexto de una asignatura de recursos humanos (en adelante RR. HH.). Cada una de las fases se puede hacer de manera consecutiva en el aula o bien se pueden llevar a cabo de manera simultánea.

I. Docente: clarificación de objetivos por parte del docente. El docente ha de tener claridad absoluta sobre los objetivos pedagógicos del debate que va a llevar a cabo. Se trata de saber si el objetivo pedagógico es conceptual (que los alumnos adquieran conocimientos de RR. HH. como por ejemplo qué es compensación), que sepan hacer algo respecto a los contenidos o procedimientos, como puede ser el caso del diseño de estrategias de retención de colaboradores (habilidad a desarrollar, un objetivo procedimental), o bien, que sean sensibles a algún asunto (objetivo sensibilizador/actitudinal hacia los recursos humanos, por ejemplo, la diversidad en la empresa o la igualdad).

2. Docente: seleccionar tema de debate. El tema de debate es distinto de la proposición de debate. Así, un tema puede ser la formación de los recursos humanos. Mientras que la resolución o proposición de debate puede ser «¿Es la formación de recursos humanos una inversión?». La elección del tema de debate puede estar a cargo de alumnos o a discreción del docente. También puede elegir el tema el docente y los alumnos formular la redacción de la proposición a debatir.

3. Docente: formar los equipos. De cara a buscar un aprendizaje verdaderamente cooperativo, el docente debe conformar equipos de debate lo más equilibrados posible. Así, se busca la heterogeneidad dentro de cada equipo, aspecto clave si buscamos una técnica auténticamente cooperativa (Pujolás, 2009). Un criterio objetivo puede ser el de asignar de manera equilibrada en cada equipo a los alumnos que hayan participado previamente en actividades de debate junto con los que no. No obstante, queda a criterio de cada docente cómo formar los equipos. En este modelo los equipos están formados por grupos de cuatro a cinco alumnos. El motivo de que se recomienden cuatro o cinco consiste en que dentro de cada debate habrá cuatro turnos de palabra por equipo. Asimismo, se recomienda que sean cinco como máximo, puesto que una de las funciones a desempeñar es la de formular preguntas al otro equipo durante los turnos de refutación y contrarrefutación. Por tanto, cuatro estudiantes pueden llevar a cabo un turno cada uno y un quinto formular las preguntas. De esta manera todos cumplen un papel activo en el aula.

4. Docente: redactar la proposición. El docente, con o sin la aprobación del alumnado, puede redactar la proposición según sus objetivos pedagógicos (ver punto i). 
Si el tema de debate es la detección del talento, se puede redactar la proposición de debate de tres maneras distintas en función de un objetivo docente u otro.

- Si el objetivo del debate es que los alumnos reflexionen sobre la causalidad de un suceso determinado, la resolución ha de ser de hecho. Esto es, la pregunta o resolución de debate ha de girar en torno a la veracidad o no de un hecho. En este caso la resolución de debate sería «La atracción de talento es el factor número uno de éxito en una empresa».

- Según el objetivo sea sensibilizar o valorar un hecho determinado, la pregunta adecuada ha ser del tipo "valoración». En estos debates se discute si un hecho cumple un determinado valor. Así, en el caso de la atracción de talento se podría preguntar ¿Cualquier método es ético para la atracción de talento?

- En caso de que el objetivo docente o pedagógico sea el de que los estudiantes creen mecanismos, desarrollen planes, propongan soluciones, etc., la resolución ha de ser de tipo político. En estas preguntas se cuestiona una solución determinada frente un problema concreto. En el tema que se expone, el ejemplo sería: ¿Se deberían emplear fórmulas de empresa privada a la hora atraer el talento en las administraciones públicas? En este caso, el equipo A favor ha de demostrar que sí se deberían emplear fórmulas de la empresa privada a la hora de atraer talento en las administraciones públicas. Por el contrario, el equipo En contra ha de demostrar, o bien que el equipo A favor se equivoca, o bien que las fórmulas habituales de las administraciones públicas son válidas para atraer talento.

Decidida la cuestión a debatir, esta se ha de comunicar a los equipos lo antes posible.

5. Alumnado: analizar la proposición. El primer logro del estudiante antes de participar en un debate es comprender qué alcance comprende la proposición. Así, el alumno habrá de analizar cada uno de los términos de la resolución. Una proposición formulada en presente o en futuro necesita una argumentación distinta. También debe abordarse de forma diferente una argumentación referente a un hecho, a una solución o a la valoración de un hecho. Cada equipo debe analizar la proposición y comenzar a generar ideas. En esta fase surgen los primeros atisbos de argumentación e ideas sobre posibles consultas de fuentes e investigación.

6. Alumnado: tempestad de ideas. Entendida la proposición, el estudiante ha de formular una tempestad de ideas sobre argumentos A favor, En contra, ideas, fuentes de datos, etcétera, o cualquier otra idea que le ayude a enfocar la preparación del debate.

7. Alumnado: investigar. Los equipos, aconsejados por el docente, han de buscar evidencias y datos que sostengan sus posturas, les ayuden a conocer el tema y construir sus primeros argumentos. Así, el docente ha de orientar hacia dónde quiere que el alumno dirija su investigación en función de determinadas fuentes. Esta fase puede durar tanto tiempo como se considere necesaria. No ha de ser en el aula necesariamente, pero sí puede hacerse un seguimiento en ella.

8. Docente: decidir y explicar el formato acorde a nuestros objetivos. No hay un formato único de debate. Sin embargo, una de las claves de éxito de un debate 
EL DEBATE COMPETITIVO EN EL AULA COMO TÉCNICA DE APRENDIZAJE COOPERATIVO EN LA ENSEÑANZA DE LA ASIGNATURA DE RECURSOS HUMANOS GUILLERMO A. SÁNCHEZ PRIETO

consiste en que los tiempos y los turnos queden claros con antelación. Estos hay que distribuirlos de manera equilibrada. En nuestro modelo y de cara a períodos lectivos de 50 minutos se recomienda que el tiempo de debate sea de media hora. Se aconseja la siguiente distribución de tiempos y turnos:

I. ${ }^{\circ}$ Exposición inicial.........

2. ${ }^{\circ}$ Exposición inicial .......

3. ${ }^{\circ}$ Refutación

4. ${ }^{\circ}$ Refutación

$5 .^{\circ}$ Contrarrefutación

6. Contrarrefutación

$7 .^{\circ}$ Conclusión.

8. ${ }^{\circ}$ Conclusión
A favor 4 minutos

En contra 4 minutos

A favor 5 minutos

En contra 5 minutos

A favor 5 minutos

En contra 5 minutos

En contra 3 minutos

A favor 3 minutos

Tiempo total: 34 minutos.

Se sigue para esta parte los criterios que Cirlin (1999: 17) reconoce como justos en un formato de debate:

- Las dos posturas tienen la misma cantidad de tiempo hablado.

- La postura A favor comienza y cierra el debate

- El formato de debate está claramente establecido con antelación para evitar sorpresas.

- El cómputo total de minutos de debate incluyendo el cambio de orador entre discurso y discurso debe evitar el aburrimiento. Así un formato ideal debería tener entre 30 y 90 minutos.

9. Docente: diseñar el acta de puntuación del debate según nuestros objetivos. El acta de debate, que funciona como una rúbrica, es la herramienta con la que el jurado decidirá qué equipo ha defendido mejor su postura. Asimismo, esta acta puede funcionar como una rúbrica para medir competencias. Así, según los objetivos docentes reflejan, podemos medir con esta acta comportamientos, conocimientos, actitudes, etc. Si, por ejemplo, el objetivo es que dominen ciertas fuentes de información sobre RR. HH. se tendrá que especificar como un apartado más del acta si utiliza o no aparato crítico a la hora de citar. Esta acta conviene que sea divulgada y explicada. En este caso el acta está adaptada a la asignatura de recursos humanos para el tema de detección de talento, pero también se tienen en cuenta aspectos propios de debate. También se puede conformar el acta y sus apartados con la opinión del alumnado.

Io. Docente: dar a conocer y explicar el acta a los equipos de debate. Es aconsejable explicar a los alumnos cada punto del acta y el porqué con una finalidad pedagógica. Así, si el estudiante conoce cuál es la intención de cada ítem y los comprende podrá ejecutarlos mejor.

II. Alumnado: preparar argumentación A favor y En contra. El docente ha de colaborar a que el alumno prepare una mínima argumentación A favor y En contra de la proposición. Cada equipo, al tener preparadas ambas posturas de la argumentación, concibe el asunto de debate de manera global. 
12. Alumnado: repartir posiciones. Una vez que el equipo ha diseñado una estrategia argumentativa, ha de decidir qué persona ocupa cada posición durante los turnos de debate. Algunos estudiantes puede que dominen mejor la exposición inicial y otros, más rápidos o experimentados en debate, sean más aptos para ocupar las posiciones de refutación. Una vez que cada estudiante conoce su rol y ha acordado junto con su equipo una estrategia argumentativa conviene que redacten sus discursos. Todo el equipo debe practicarlos hasta el momento en que se considere que el debate está suficientemente preparado.

13. Docente: orientar a los equipos en pautas mínimas de comunicación. Es aconsejable proporcionar a los participantes algunas pautas para comunicar en público y defender argumentos, así como la mecánica del debate (qué es una exposición inicial, la finalidad de una refutación, etc.), qué se espera de ellos en cada parte del debate. Quizás no sea necesaria esta orientación en oratoria si se cuenta ya con una asignatura o formación al respecto.

14. Docente: escoger jurados. Para todo debate es necesario un jurado. Este jurado se puede elegir entre los alumnos. La idea del modelo está en que todos los alumnos hagan de debatientes y todos hagan el papel de jueces. En el modelo propuesto, cualquiera de los equipos que no esté debatiendo puede hacer el papel de jurado. En este jurado se puede integrar al docente o no. El jurado no sólo ha de utilizar el acta como medio para designar un vencedor, sino también como herramienta para recabar comentarios que mejoren la competencia que hayamos especificado.

15. Docente: organizar el espacio del aula. Para que se pueda practicar al máximo posible la expresión oral del alumnado, conviene configurar el espacio del aula, como se explica en el Gráfico r. El orador en uso de la palabra se colocará de pie junto al atril o en el espacio que se designe para ello. El aula debe organizarse de manera que el equipo A favor y En contra estén enfrentados. Frente a ambos equipos se situará el jurado formado por docente y/o alumnos y detrás de ellos el público. El público puede tener también un rol determinado y activo a la hora de emitir su voto.

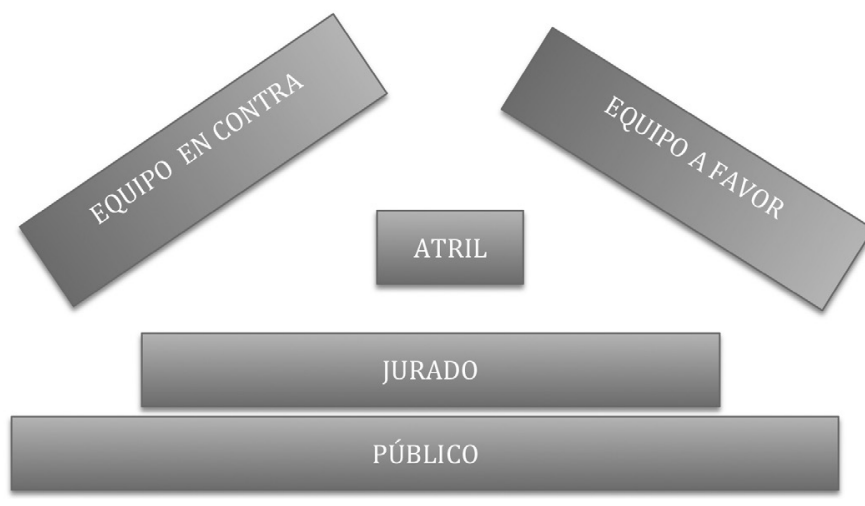

Figura i. Configuración espacial del aula en el modelo de debate. Fuente: Elaboración propia. 
EL DEBATE COMPETITIVO EN EL AULA COMO TÉCNICA DE APRENDIZAJE COOPERATIVO EN LA ENSEÑANZA DE LA ASIGNATURA DE RECURSOS HUMANOS GUILLERMO A. SÁNCHEZ PRIETO

16. Docente: sorteo de posturas. Antes de cada debate, con la antelación que considere el docente, se sorteará la postura que ha de defender cada equipo. Así, ambos equipos deben llevar preparada tanto la postura A favor como la postura En contra. Si queremos que se tenga una visión holística del tema de debate, es conveniente que los equipos se preparen A favor y En contra. Si el objetivo docente no es un conocimiento tan amplio no será necesario que preparen ambas posturas. Queda a discreción del docente que las posturas se sorteen con mayor o menor antelación al mismo.

\subsection{Ejecución del debate}

En esta parte el docente apenas interviene pues el debate se autorregula con el uso de los turnos consensuados. En todo caso, conviene que un alumno o el docente ejerza una función de control para garantizar el cumplimiento de tiempos. En caso de que un debatiente exceda su tiempo de alocución, el docente o el alumno encargado ha de tenerlo en cuenta y restringir el turno de palabra o reflejarlo en la puntuación mediante una puntuación menor, por ejemplo.

A continuación se detalla qué se debe hacer en cada uno de los turnos según el formato expuesto previamente.

- Exposición inicial. El debatiente encargado del turno debe explicar cuáles son los argumentos principales que sostienen su respuesta, A favor o En contra. El equipo A favor debe exponer por qué es cierta la resolución. El equipo En contra debe demostrar que la resolución es falsa. Si tratamos el caso de la gestión del talento y seguimos la proposición «La gestión del talento marca la diferencia en las empresas de éxito», el equipo A favor ha de demostrar que la gestión del talento es la esencia causal del éxito empresarial. El equipo En contra tiene dos posibilidades:

a) Demostrar que la gestión del talento no es la clave del éxito en las empresa, con lo cual debe demostrar qué factores causan el éxito empresarial, financiero, comercial, estratégico, etc.

b) Demostrar que el equipo A favor está equivocado pues no defiende de manera correcta su postura.

- Refutaciones y contrarrefutaciones. Estos dos son los apartados que podríamos considerar más propios de un debate y que, por tanto, confieren su característica más singular a esta forma de intercambio dialéctico. Así, en refutaciones y contrarrefutaciones es cuando se permite formular interpelaciones o preguntas al orador que está en uso de la palabra. Este decidirá si admite las preguntas del equipo rival. El propósito de una refutación consiste en hacer ver al jurado los errores de la argumentación contraria. En las contrarrefutaciones se ha de contraatacar los ataques recibidos. Las exposiciones que estos oradores llevan a cabo han de ser diseñadas dependiendo del discurso del equipo contrario, por lo que no han de llevarse preparadas, sino que han de ser formuladas in situ.

- Conclusión. El turno de conclusiones es, como su nombre indica, el momento de confirmar si la defensa de la postura ha sido correcta. A su vez, es el momento en el que se exhorta al jurado a concederle su voto. No se trata de refutar o contrarrefutar ni, por supuesto, de mostrar evidencias nuevas. 


\subsection{Análisis y juicio del debate}

Docente y alumnado: juzgar el debate. Profesorado o alumnado pueden juzgar el debate. En esta parte el jurado o el profesorado sólo intervendrá en caso de considerarlo necesario: extensión excesiva en intervenciones, desórdenes, imprevistos, etc. En el modelo presentado recomendamos que se confiera la mayor autoridad posible al alumnado no debatiente.

El jurado ha de juzgar el debate según los criterios que hemos visto, quedando patentes sus criterios en el acta o rúbrica. Además, puede resultar pedagógico que emitan una valoración verbal cualitativa pudiendo evaluar comportamientos a modo de feedback.

El jurado ha de valorar el debate y dar una devolución del desempeño a los participantes de manera individual o como equipo. Los comentarios que se formulan no justifican el veredicto del jurado pues tienen un fin puramente constructivo.

Docente: calificación. El docente puede calificar en función del acta a modo de rúbrica. Queda a criterio del docente ponderar el peso de los ítems del acta según sus objetivos docentes. El ejemplo propuesto muestra una estructura de acta o rúbrica diseñada para evaluar el debate sobre la proposición ¿Es la gestión de talento la clave del éxito empresarial?

Tabla i. Acta de items de juez de Debate

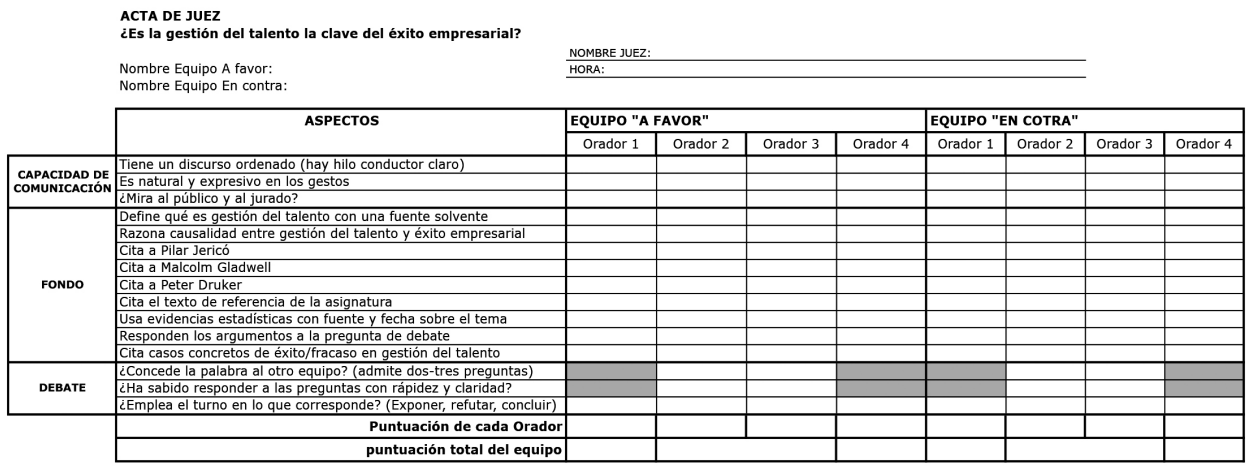

EQUIPO GANADOR:

INSTRUCCIONES

Sil la respuesta es afirmativa se marcará una x en la casilla del orrader que haya curnplida con cada aspecto.

Tras la valoracación se sumarán el total de respuestas afirmativas.

Fuente: Elaboración propia inspirada en SÁnCHEz PRIETO (2007).

\section{Consonancia del debate con las metodologías activas}

El enfoque que se da a esta parte consiste en la definición y explicitación de las características del aprendizaje cooperativo (en adelante Ac) y comprobar en qué medida se ajustan al método del debate competitivo y sus características propias. 
EL DEBATE COMPETITIVO EN EL AULA COMO TÉCNICA DE APRENDIZAJE COOPERATIVO EN LA ENSEÑANZA DE LA ASIGNATURA DE RECURSOS HUMANOS GUILLERMO A. SÁNCHEZ PRIETO

El aprendizaje cooperativo se encuadra dentro de las metodologías activas. Según Benito y Cruz (2003: 22), las tres principales metodologías activas son: el aprendizaje cooperativo, el aprendizaje basado en problemas y el método del caso. El objetivo de este trabajo consiste en demostrar que el debate es una herramienta propia de las metodologías activas y en concreto un modelo de aprendizaje cooperativo. Así, se enuncian las características de las tres principales estrategias de las metodologías activas y veremos en qué medida el modelo de debate en el aula las cumple o no.

TABLA 2. Características de las metodologías activas y su presencia en el modelo de debate

\begin{tabular}{|c|c|c|}
\hline METODOLOGÍA & CARACTERÍSTICAS & PRESENTE EN DEBATE \\
\hline \multirow[t]{4}{*}{$\begin{array}{l}\text { Aprendizaje } \\
\text { cooperativo }\end{array}$} & $\begin{array}{l}\text { Construcción de conocimiento conjunto entre } \\
\text { profesores y equipos de alumnos }\end{array}$ & Sí \\
\hline & Promueve la responsabilidad personal & Sí \\
\hline & Responsabilidad compartida & Sí \\
\hline & Habilidades interpersonales & Sí \\
\hline \multirow{4}{*}{$\begin{array}{l}\text { Aprendizaje basado } \\
\text { en problemas }\end{array}$} & Alumnos en grupo & Sí \\
\hline & De forma autónoma & Sí \\
\hline & Encontrar respuesta a pregunta o problema & Depende del tipo de resolución \\
\hline & Buscar, entender e integrar conceptos & Sí \\
\hline \multirow[t]{6}{*}{ Método del caso } & Trabajar sobre situación real & Sí \\
\hline & Tiene que ver con decisión u oportunidad & Sí \\
\hline & Uso del conocimiento & Sí \\
\hline & Problema o cuestión compleja & Sí \\
\hline & Analizar información & Sí \\
\hline & Posicionarse, experimentar, tomar decisiones & Sí \\
\hline
\end{tabular}

Fuente: Elaboración propia a partir de BENITO y CRUZ (2003).

A continuación, se comprueba por qué cada una de sus características está presente o no en nuestro modelo de debate.

I. Constructivismo: construcción de conocimiento compartido entre profesores y equipos de alumnos. En debates sí se da esta característica, puesto que tanto alumnos como docentes pueden sugerir preguntas o resoluciones de debate. Además, ambas partes sugieren ideas y diferentes visiones sobre preguntas comunes. Así, cuando un profesor sugiere tratar un determinado tema, los alumnos pueden hacer propuestas alternativas. Del mismo modo, cuando docentes y alumnos han de valorar si los datos son relevantes o no para el debate, lo hacen todos juntos.

2. Promueve la responsabilidad personal. A la hora de participar en un debate y a la hora de comunicar frente al aula, el alumno ha de cumplir un papel en el que ha de afrontar, en solitario, una responsabilidad. El debatiente, aunque respaldado por un equipo, es el que ha de tomar la palabra y defender unos argumentos frente a una audiencia.

3. Responsabilidad compartida. Tanto para preparar un debate como para juzgarlo, todo el equipo es responsable de un buen resultado. Si un debatiente no lleva preparado mínimamente su discurso, queda en evidencia por no cumplir con su 
EL DEBATE COMPETITIVO EN EL AULA COMO TÉCNICA DE APRENDIZAJE COOPERATIVO EN LA ENSEÑANZA DE LA ASIGNATURA DE RECURSOS HUMANOS GUILLERMO A. SÁNCHEZ PRIETO

labor y el resto del equipo por no prever ese fallo. Si el jurado está formado por alumnos, estos han de emitir un veredicto sobre qué equipo es ganador y evaluar el desempeño de los debatientes con recomendaciones de mejora. Todas las responsabilidades se comparten.

4. Habilidades interpersonales. En un debate se deben utilizar habilidades como la oratoria, la dialéctica, la persuasión, etc. Antes de un debate, durante la preparación, se han de emplear el trabajo en equipo, la toma de decisiones grupales, gestión de conflictos, etc. Todo estrechamente relacionado con la comunicación y las habilidades interpersonales.

5. Trabajo en equipo. En el formato de debate de este modelo se enfrenta un equipo frente a otro. Se aprende de manera conjunta antes del debate pues se trabaja con iguales. También se aprende en equipo durante el debate con los propios y los competidores pues las posturas opuestas se necesitan para discutir y, por tanto, aprender. Asimismo, después del debate el jurado también colabora en ese aprendizaje al dar retroalimentación con su evaluación del desempeño.

6. Autonomía. En un debate el alumno decide qué aprender dentro de unos límites dados por la pregunta y el acta de debate. No se tiene por qué dirigir necesariamente al alumnado, por ejemplo, cómo estudiar los planes de carrera en la empresa ni qué fuentes ha de consultar, pero sí se le recomendará cómo abordar un acercamiento a los planes de carrera como elemento clave del desarrollo de los RR. HH. y qué tipo de fuentes son más rigurosas al respecto. No obstante, el hecho de elegir una u otra fuente o decidir qué enfoque da a los planes de carrera será decisión de los propios alumnos como debatientes.

7. Encontrar respuesta a preguntas o problemas. Quizás sea esta la característica más discutible sobre si un debate es o no una metodología activa. En realidad, en un debate no se llega a encontrar una respuesta definitiva pues siempre hay un A favor o un En contra y ninguno de los dos da la razón al otro en el debate. De esta manera, no se llega a resolver el problema que se plantea puesto que lo que se persigue es, sobre todo, la reflexión y discusión de una cuestión.

8. Buscar entender e integrar conceptos. En cualquier debate se debe discutir siempre la naturaleza del concepto sobre el que se está debatiendo. Así, si se debate sobre capitalismo, se tendrá que tener una definición rigurosa de qué es capitalismo. Por otra parte, una vez que se entiende el concepto de capitalismo se ha de integrar dentro del entorno del debate y responder a la pregunta de debate de manera global.

9. Trabajar sobre una situación real. En un debate en el que se trate una cuestión histórica, científica o conceptual siempre se está trabajando sobre situaciones reales. Si se discute «¿Es necesaria una reinvención del capitalismo?», el capitalismo es un concepto real. Por ejemplo, se puede debatir sobre si el capitalismo es positivo para el desarrollo de la persona o si el capitalismo fue efectivo en la salida de la depresión económica del 29.

Io. Tiene que ver con decisión u oportunidad. En el presente modelo la decisión u oportunidad no está en el sentido de elección, pero sí tiene que ver con la capacidad que el alumno desarrolla al tomar sus decisiones sobre la argumentación que emplee en el debate. Así, si un alumno tiene que defender que el sector público es capaz de revitalizar una economía, el alumno ha de tomar sus propias decisiones sobre su estrategia argumentativa o sobre qué fuentes consulta, pero siempre teniendo en cuenta al equipo. 
II. Uso del conocimiento. En cualquier debate el alumno ha de emplear el conocimiento que previamente ha recolectado al tener que buscar evidencias. El hecho de buscar evidencias de autoridad no es solamente para que el alumno sea capaz de encontrarlas, sino para que las emplee como herramienta de persuasión hacia el jurado. Así, se pasa del conocimiento inerte al significativo.

I2. Problemas o cuestiones complejas. En cualquier debate se ha de plantear siempre un problema o una cuestión a resolver que, según el perfil del alumnado (edad, curso académico, etc.), podrá ser más o menos complejo.

13. Analizar información. El alumno ha de discriminar y decidir qué información usa, pues no toda la información resulta pertinente a la pregunta o puede ser útil a la hora de argumentar. Asimismo, no todas las informaciones o evidencias tienen la misma fuerza probatoria o persuasiva de cara a un jurado.

I4. Posicionarse, experimentar y tomar decisiones. El participante ha de posicionarse A favor o En contra de la pregunta, con lo que no necesariamente ha de estar de acuerdo respecto sus convicciones personales. Por ejemplo, si un alumno debe defender un Expediente de Regulación de empleo como mejor forma de salir de una crisis financiera en una empresa no implica que necesariamente concuerde personalmente con esto. Esta complejidad obliga al alumno a pensar de manera más abierta y crítica.

\section{El debate competitivo como técnica de aprendizaje cooperativo}

Vistas las consonancias con el debate en el aula y las metodologías activas se expondrá a continuación lo propio para el aprendizaje cooperativo y el debate en el aula.

En primer lugar, es necesario explicar qué se entiende por aprendizaje cooperativo y al mismo tiempo considerar sus consonancias o disonancias con el modelo de debate en el aula propuesto. Así, se agrupan las definiciones más relevantes de aprendizaje cooperativo, observando si cumplen la definición del modelo propuesto.

TABLA 3. Características comunes y particulares del aprendizaje cooperativo dentro del debate

\begin{tabular}{|l|l|l|}
\hline AUTORES & $\begin{array}{l}\text { Características comunes del } \\
\text { concepto de AC }\end{array}$ & $\begin{array}{l}\text { Explicación de por qué el debate cumple o no la } \\
\text { característica }\end{array}$ \\
\cline { 2 - 3 } & Trabajo en grupo & $\begin{array}{l}\text { Los debatientes pueden organizarse en equipos de 4-5 par- } \\
\text { ticipantes o individualmente, pero siempre necesitan a otro } \\
\text { con el que debatir, con lo cual siempre será en grupo. }\end{array}$ \\
\cline { 2 - 3 } & $\begin{array}{l}\text { Todos los miembros del gru- } \\
\text { po se involucran en el apren- } \\
\text { dizaje de todos los demás }\end{array}$ & $\begin{array}{l}\text { Al participar unos con otros y unos frente a otros, en el } \\
\text { intercambio dialéctico, todos los miembros del grupo se } \\
\text { han de involucrar necesariamente. }\end{array}$ \\
\cline { 2 - 3 } & $\begin{array}{l}\text { Participación de todos los } \\
\text { estudiantes }\end{array}$ & $\begin{array}{l}\text { Todos los participantes han de debatir en la clase y todos } \\
\text { han de juzgar por lo que se implica a todo el grupo. }\end{array}$ \\
\cline { 2 - 3 } & $\begin{array}{l}\text { Variedad de actividades de } \\
\text { aprendizaje }\end{array}$ & $\begin{array}{l}\text { En la preparación del debate se ha de investigar, pensar } \\
\text { en tesis, argumentos, refutaciones y extraer conclusiones. } \\
\text { Asimismo, la puesta en escena frente al resto de la clase } \\
\text { hace imprescindible comunicar en público, evaluar el } \\
\text { desempeño de los compañeros y redactar discursos. }\end{array}$ \\
\hline
\end{tabular}


EL DEBATE COMPETITIVO EN EL AULA COMO TÉCNICA DE APRENDIZAJE COOPERATIVO EN LA ENSEÑANZA DE LA ASIGNATURA DE RECURSOS HUMANOS GUILLERMO A. SÁNCHEZ PRIETO

\begin{tabular}{|c|c|c|}
\hline & $\begin{array}{l}\text { Características particulares } \\
\text { del concepto de AC de cada } \\
\text { autor }\end{array}$ & \\
\hline $\begin{array}{l}\text { Johnson, } \\
\text { Johnson } \\
\text { y Holubec }\end{array}$ & $\begin{array}{l}\text { - Grupos reducidos } \\
\text { - Trabajo para aprendizaje } \\
\text { de los demás }\end{array}$ & $\begin{array}{l}\text { Cada equipo de debate suele constar de un numero de } \\
\text { cuatro a cinco debatientes. En cada debate, por tanto } \\
\text { participan de ocho a diez estudiantes. } \\
\text { Al ser un trabajo en equipo, la investigación y la prepara- } \\
\text { ción previa necesita del trabajo de todo el grupo de debate } \\
\text { en mayor o menor medida. }\end{array}$ \\
\hline $\begin{array}{l}\text { Slavin, } \\
\text { Serrano, } \\
\text { González } \\
\text { Fernández } \\
\text { y García } \\
\text { Ruiz }\end{array}$ & $\begin{array}{l}\text { - Concepción holística del } \\
\text { proceso de enseñanza } \\
\text { - Método alternativo de } \\
\text { organización de procesos } \\
\text { cognitivos dentro y fuera } \\
\text { del aula }\end{array}$ & $\begin{array}{l}\text { El debate mezcla los contenidos educativos con las habili- } \\
\text { dades sociales. } \\
\text { El participante se ve obligado a buscar evidencias con el } \\
\text { fin de sostener argumentos y buscar ideas ocurrentes para } \\
\text { captar la atención a la hora de ejecutar los discursos den- } \\
\text { tro y fuera del aula. }\end{array}$ \\
\hline Trujillo & $\begin{array}{l}\text { Construcción participativa } \\
\text { del conocimiento }\end{array}$ & $\begin{array}{l}\text { El participante ha de participar al afirmar o negar la reso- } \\
\text { lución de debate. }\end{array}$ \\
\hline $\begin{array}{l}\text { Fathman } \\
\text { y Kessler }\end{array}$ & $\begin{array}{l}\text { Evaluación de forma indi- } \\
\text { vidual }\end{array}$ & $\begin{array}{l}\text { El modelo permite la evaluación individual y de equipo al } \\
\text { mismo tiempo. }\end{array}$ \\
\hline $\begin{array}{l}\text { Eggen } \\
\text { y Kauchak }\end{array}$ & Incrementa el liderazgo & $\begin{array}{l}\text { El hecho de tomar la palabra y ser protagonista del proce- } \\
\text { so de aprendizaje obliga a incrementar las capacidades de } \\
\text { liderazgo. }\end{array}$ \\
\hline $\begin{array}{l}\text { Johnson, } \\
\text { Johnson } \\
\text { y Stanne }\end{array}$ & $\begin{array}{l}\text { Diversidad de métodos de } \\
\text { enseñanza }\end{array}$ & \\
\hline
\end{tabular}

Fuente: Elaboración propia.

Según Benito y Cruz (2005: 24-25) citan a Smith (1996: 7I-82), algunas de las características del aprendizaje cooperativo son la interdependencia positiva, la evolución individualizada y la responsabilidad personal, frecuente interacción cara a cara, uso adecuado de destrezas interpersonales y grupales, revisión periódica del proceso del grupo entre otras.

A continuación, se explica cada una de ellas y qué relación tienen con nuestro modelo de debate en el aula.

- Interdependencia positiva: «Todos los integrantes están obligados a confiar en los otros para conseguir el objetivo. Si uno falla en su parte, todos sufren las consecuencias. El éxito de cada cual depende del éxito de los demás» (Benito y Cruz, 2005: 24 y Smith). En el modelo propuesto, cada equipo depende de cómo cada uno de los debatientes desempeñe su papel y de cómo el equipo, como tal, lleve a cabo su misión. Así, si un debatiente no cumple con su papel y su desempeño es pobre, esto afecta al resultado y a la nota del equipo.

- «Evaluación individualizada y responsabilidad personal: el aprendizaje cooperativo incorpora siempre la evaluación individual además de la grupal» (Benito y Cruz, 2005: 24). Gracias al acta de debate del modelo propuesto, cada alumno puede obtener una calificación o evaluación y el equipo también obtiene la misma, de manera que el desempeño individual afecta al resultado del equipo (Tabla I). 
EL DEBATE COMPETITIVO EN EL AULA COMO TÉCNICA DE APRENDIZAJE COOPERATIVO EN LA ENSEÑANZA DE LA ASIGNATURA DE RECURSOS HUMANOS GUILLERMO A. SÁNCHEZ PRIETO

- Frecuente interacción cara a cara, «aunque una parte del trabajo debe ser realizado individualmente, otra parte sólo se puede dar de forma interactiva. Supone razonar sobre cómo resolver los problemas, explicar un determinado concepto a los demás, asegurarse de que lo han entendido, conectando con el trabajo presente» (Benito y Cruz, 2005: 24). En el modelo propuesto resulta evidente la interacción cara a cara, pero no solo durante el debate, sino también antes, resultando imprescindible la interacción y el aprendizaje mutuo, así como la enseñanza de unos a otros. Se hace esencial el manejo de conceptos y su reflexión para poder realizar un desempeño mínimo en el debate (tempestad de ideas, contraste de argumentos, práctica de discursos, etc.). Asimismo, hay una parte de trabajo y aprendizaje que se ha de llevar a cabo individualmente (investigación, redacción de discursos, etc.).

- Uso adecuado de destrezas interpersonales y grupales: «Los estudiantes deben adoptar un doble compromiso con la tarea (aprendizaje del tema académico) y con el trabajo de equipo de manera efectiva como grupo» (Benito y Cruz, 2005: 24). El alumno ha de investigar, comprender conceptos, manejar datos de cara al debate, lo cual le lleva a conocer el tema académico de debate en una mínima profundidad. Los alumnos, por otra parte, han de trabajar en conjunto, en mayor o menor grado. Así, tendrán que resolver conflictos, tomar decisiones de manera conjunta y aprenderán en equipo.

- «Revisión periódica del proceso del grupo. Los miembros del grupo periódicamente revisan el cumplimiento de tareas, identifican los problemas del grupo, deciden los cambios pertinentes» (Benito y Cruz, 2005: 24). A la hora de preparar el debate, el equipo ha de hacer necesariamente seguimiento de la investigación de los logros alcanzados así como de los no alcanzados. Lo cual les lleva necesariamente a tomar decisiones sobre qué han de hacer y qué estrategia seguir.

Se puede comprobar que el debate cumple al menos las características mínimas del aprendizaje cooperativo.

\section{Conclusiones}

I. El modelo propuesto se enmarca claramente dentro de las metodologías activas al cumplir con la mayor parte de sus características.

2. El modelo de debate en el aula se puede considerar conceptualmente en el marco del aprendizaje cooperativo.

3. El modelo permite la calificación individual y la colectiva.

4. Queda por demostrar en qué tipo de disciplinas o áreas de conocimiento es más propio el debate cooperativo.

5. Queda por investigar la valoración que hacen de esta técnica docentes y alumnos al margen de su eficacia frente a otras alternativas de evaluación y de aprendizaje.

\section{Bibliografía}

Авніліт, R. у Mассніетте, B. (2005) Debating the Issues: A Tool for Augmenting Critical Thinking Skills of Marketing Students. Journal of Marketing Education, vol. 27, n. ${ }^{\circ}$ 3, 264-276. 
Bellon, J. (2000) A research-based justification for debate across the curriculum. Argumentation and Advocacy, vol. 36, I67I-175.

Benito, A. y Cruz, A. (2005) Nuevas claves para la docencia universitaria en el espacio europeo de educación superior. Madrid, España: Ed. Narcea.

Brenifier, O. (2005) Enseñar mediante el debate. Ciudad de México, México: Ed. Édere.

Cattani, A. (2003) Los usos de la retórica. Madrid, España: Alianza Ensayo.

Cirlin, A. (1999) Academic debate and program development for students and teachers around the world. An introductory textbook, handbook and sourcebook. San Diego, Estados Unidos de América: Isocratic Press.

EgGen, P. y KauchaK, D. (1999) Estrategias docentes: Enseñanza de contenidos curriculares y desarrollo de habilidades de pensamiento. Ciudad de México, México: Fondo de Cultura Económica.

Fathman, A. K. y Kessler, C. (1993) Cooperative language learning in school contexts. Annual Review of Applied Linguistics, I3, I27-I40.

González Fernández, N. y García Ruiz, M. R. (2007) El aprendizaje cooperativo como estrategia de Enseñanza-Aprendizaje en Psicopedagogía. Revista Iberoamericana de Educación, $\mathrm{n}^{\circ}{ }^{\circ} 42 / 6, \mathrm{I}-\mathrm{I} 3$.

Huber, R. y Snider, A. (2006) Influencing through argument. Nueva York, Estados Unidos de América: Idebate press.

Johnson, D. W.; Johnson, R. T. y Holubec, E. J. (1999) El aprendizaje cooperativo en el aula. Buenos Aires, Argentina: Ed. Paidós.

Mitchell, G. (1998) Pedagogical possibilities for argumentative agency in academic debate. Argumentation E Advocacy, vol. 35, Issue 2, 4I-60.

Pujolás, P. (2009) 9 ideas clave del aprendizaje cooperativo. Barcelona, España: Ed. Graó.

SÁnchez Prieto, G. (2007) Educar en la palabra. Manual de argumentación, oratoria y debate. Madrid: Ed. Fundación Universitaria Española.

Serrano, J. M. (1996) El aprendizaje cooperativo. Murcia, España: Caja Murcia Obra Social.

Slavin, R. (1999) Aprendizaje cooperativo: Teoría, investigación y práctica. Buenos Aires, Argentina: Aique.

Smith, K. (1996) Making Groupwork work. En Sutherland y Boncel (eds.) Using active learning in college clases: a range of options for faculty. New directions for Teaching and Learning, n. ${ }^{\circ}$ 67, Fall, 7I-82.

Takanokura, M. y Hayashi, S. (2008) Educational Effects of Practical Education Using a Debate Exercise on Engineering Ethics. Journal of Japanese Society for Engineering Education, volume 56, Issue 2, 2_8-2_13.

Trujillo, F. (2006) Aprendizaje cooperativo para la enseñanza de la lengua. Granada, España: Grupo Editorial Universitario Universidad de Granada.

Yasuko, K. (2003) Effects of Introduction of Debating Techniques in a Maternal Nursing Course. Bulletin of Shimane Medical University, vol. 26, II-I7. 